\title{
Elementary abelian operator groups
}

\section{Fletcher Gross}

\author{
Suppose $G$ is a finite solvable $p^{\prime}$-group admitting the \\ elementary abelian $p$-group $A$ as an operator group. If \\ $n=\max \left\{\right.$ nilpotent length of $\left.C_{G}(X) \mid X \in A^{\#}\right\}$ and $|A| \geq p^{n+2}$, \\ then the nilpotent length of $G$ is $n$.
}

\section{Introduction}

Suppose $A$ is an elementary abelian $p$-group of order $p^{m}$ acting as an operator group on the finite $p^{\prime}$-group $G$. If $m \geq 3$ and $C_{G}(X)$ is nilpotent for each non-identity element $X$ in $A$, then Ward [8] showed that $G$ is nilpotent. More recently, Ward [9] proved that if $G$ is solvable, $m \geq 4$, and the derived group of $C_{G}(X)$ is nilpotent for each non-identity element $X$ in $A$, then $G^{\prime}$ is nilpotent. The principal result (Theorem 3.1) of the present paper asserts that if $G$ is solvable, $n$ is the maximum of the nilpotent lengths of $C_{G}(X)$ where $X$ runs through the non-identity elements of $A$, and $m \geq n+2$, then the nilpotent length of $G$ is $n$. Using this result, an easy argument shows that if $G$ is solvable, $C_{G}(X)$ is supersolvable for each non-identity element $X$ in $A$, and $m \geq 4$, then $G$ is super-solvable. Examples are given showing the necessity of the inequalities $m \geq n+2$ and $m \geq 4$ in these results.

These theorems depend on a rather complicated technical result (Theorem 2.4) proved in $\$ 2$ about the upper nilpotent series of a finite solvable group $G$ which admits an operator group $A$ where

Received 15 February 1972. The research was supported in part by a grant from the ivational Science Foundation of the USA. 
$(|G|,|A|)=1$. The main results are proved in $\$ 3$ and examples are given in $\$ 4$.

\section{Notation and preliminary results}

All groups considered in this paper are finite. If $G$ is a group, $F_{0}(G)=1$ and $F_{n+1}(G) / F_{n}(G)=F\left(G / F_{n}(G)\right)$ equals the largest normal nilpotent subgroup of $G / F_{n}(G)$. If $G$ is solvable, $Z(G)$ is the smallest non-negative integer $n$ such that $F_{n}(G)=G$. The rest of the notation agrees with [2]. We now prove a number of technical results needed for the main theorems.

THEOREM 2.1. Suppose $P$ is a p-group which admits the group $G$ as an operator group. Assume $Q$ is a normal $p^{\prime}$-subgroup of $G$ which centralizes every $G$-invariant proper subgroup of $P$ but $[P, Q] \neq 1$. Then $P$ is a special $p$-group and any proper G-irvariant subgroups of $P$ are contained in $P^{\prime}$.

Proof. This follows immediately from Theorem $C$ of [6].

LEMMA 2.2. Let $P$ be a p-subgroup of the group $G$. Assume $P \leq E_{2}(G)$ but $P \neq F_{1}(G)$. Then for some prime $q \neq p$, the sylow q-subgroup of $F_{1}(G)$ is not centralized by $P$.

Proof. Let $H$ be a Hall $p^{\prime}$-subgroup of $E_{1}(G)$. If $S$ is a Sylow p-group of $E_{2}(G)$, then $H C_{S}(H)$ is a normal nilpotent subgroup of $G$. Hence $C_{S}(H) \leq E_{1}(G)$. This implies that $[H, P] \neq 1$. Since $H$ is nilpotent, the desired result follows immediately.

LEMMA 2.3. Let $G$ be a solvable group and $H$ a subgroup of $G$. Assume that $P_{1}, \ldots, P_{n}(n>1)$ are subgroups of $H$ and $p_{1}, \ldots, p_{n}$ are primes satisfying the following conditions:
(a) $P_{i}$ is a $p_{i}$-group if $1 \leq i \leq n$;
(b) $p_{i} \neq p_{i+1}$ if $1 \leq i \leq n-1$;
(c) $P_{i+1}<N_{G}\left(P_{i}\right)$ if $1 \leq i \leq n-1$; 
(d) $P_{i} \leq F_{i}(G)$ if $1 \leq i \leq n$;

(e) $\left[P_{n}, P_{n-1}, \ldots, P_{2}, P_{1}\right] \neq 1$.

Then $Z(H) \geq n$.

Proof. Clearly $F_{i}(H) \geq F_{i}(G) \cap H \geq P_{i}$ for $1 \leq i \leq n$. Suppose $Z(H)<n$. Then $F_{n-1}(H)=H$. Since $H / F_{n-2}(H)$ is nilpotent and $\left(\left|P_{n}\right|,\left|P_{n-1}\right|\right)=1$, we obtain $\left[P_{n}, P_{n-1}\right] \leq E_{n-2}(H) \cap P_{n-1}$. Now $P_{n-1}$ normalizes $P_{n-2}$ and $P_{n-2}$ is a $P_{n-1}^{\prime}$-subgroup of $E_{n-2}(H)$. Thus $\left[P_{n}, P_{n-1}, P_{n-2}\right] \leq F_{n-3}(H) \cap P_{n-2}$. Continuing in this way, we eventually obtain

$$
\left[P_{n}, P_{n-1}, \ldots, P_{2}, P_{1}\right] \leq E_{0}(H) \cap P_{1}=1
$$

which is a contradiction. Thus $Z(H) \geq n$.

THEOREM 2.4. Suppose $A$ is an operator group on the solvablè group $G$ where $(|A|,|G|)=1$. Assume $Z(G)=n>0$. Then there are primes $p_{1}, \ldots, p_{n}$ and A-invariant subgroups $P_{1}, \ldots, P_{n}$ in $G$ such that:

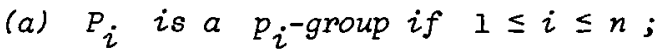

(b) $p_{i} \neq p_{i+1}$ if $1 \leq i \leq n-1$;

(c) $P_{i} \leq N_{G}\left(P_{j}\right)$ if $1 \leq j \leq i \leq n$;

(d) $P_{i} \leq F_{i}(G)$ but $P_{i} \ddagger F_{i-1}(G)$ if $1 \leq i \leq n$;

(e) $\left[P_{i+1}, P_{i}\right]=P_{i}$ if $1 \leq i \leq n$;

(f) if $Q$ is an A-invariant proper subgroup of $P_{n}$, then $Q \leq F_{n-1}(G)$

(g) if $I \leq i \leq n-1$ and $Q$ is a proper subgroup of $P_{i}$ which is invariant under $A \prod_{i<j} P_{j}$, then $\left[P_{i+1}, Q\right] \leq E_{i-1}(G)$.

Proof. If $n=1$, simply let $P_{1}$ be a minimal $A$-invariant subgroup of $G$. Suppose next $n=2$. Let $p_{2}$ be a prime dividing $\left|G / \vec{E}_{1}(G)\right|$. 
By [3, Corollary 2, p. 124], there is an $A$-invariant Sylow $p_{2}$-subgroup $P$ in $G$. Let $P_{2}$ be minimal with respect to: $P_{2} \leq P, P_{2} \neq F_{1}(G)$, and $P_{2}$ is $A$-invariant. By Lemma 2.2, there is a prime $p_{1} \neq p_{2}$ such that $P_{2}$ does not centralize the Sylow $p_{1}$-subgroup of $F_{1}(G)$. Choose $P_{1}$ to be minimal with respect to: $P_{1}$ is a $P_{1}$-subgroup of $F_{1}(G)$, $N_{G}\left(P_{1}\right) \geq P_{2}, P_{1}$ is $A$-invariant, and $\left[P_{2}, P_{1}\right] \neq 1$. Since $\left[P_{1}, P_{2}, P_{2}\right]=\left[P_{1}, P_{2}\right]$ from [3] and $\left[P_{1}, P_{2}\right]$ is A-invariant and normalized by $P_{2}$, we must have $\left[P_{2}, P_{1}\right]=P_{1}$. This proves the theorem for $n \leq 2$. We now assume $n>2$ and proceed by induction on $n$.

By [3], there is an A-invariant Carter subgroup $C$ of $F_{2}(G)$. Let $N=N_{G}(C) \cdot N \cap E_{2}(G)=C$ and, by the Fitting argument, $G=E_{2}(G) N$.

Since $F_{2}(G) / E_{1}(G)$ is nilpotent, $F_{2}(G)=F_{1}(G) C$. Suppose now $2 \leq i \leq n$. Then from $G=E_{2}(G) N$ follows $E_{i}(G)=F_{2}(G)\left(E_{i}(G) \cap N\right)$. Since $Z\left(E_{i}(G) / E_{2}(G)\right)=i-2$ and $E_{i}(G) \cap N \cap F_{2}(G)=C \leq F_{1}(N)$, we find that $Z\left(F_{i}(G) \cap N\right) \leq i-1$. Hence $F_{i}(G) \cap N \leq E_{i-1}(N)$. Conversely, $E_{i-1}(N) F_{2}(G)$ is normal in $N F_{2}(G)=G$ and

$$
l\left(F_{i-1}(N) F_{2}(G)\right)=Z\left(F_{i-1}(N) C F_{1}(G)\right)=2\left(F_{i-1}(N) F_{1}(G)\right) \leq i .
$$

This implies that $F_{i-1}(N) \leq F_{i}(G) \cap N$. Hence $F_{i}(G) \cap N=F_{i-1}(N)$ for $2 \leq i \leq n$. A consequence of this is that $l(N)=n-1$.

By induction, there are primes $q_{1}, \ldots, q_{n-1}$ and $A$-invariant subgroups $Q_{1}, \ldots, Q_{n-1}$ in $N$ satisfying (a) through (g) for $N$. For $3 \leq i \leq n$, let $p_{i}=q_{i-1}$ and $P_{i}=Q_{i-1}$. From the fact that $F_{j}(G) \cap N=F_{j-1}(N)$ for $2 \leq j \leq n$, it follows that $P_{3}, \ldots, P_{n}$ satisfy the required conditions with respect to $G$. It remains to chose $P_{1}$ and $P_{2}$.

Now $P_{3} \leq F_{2}(N) \leq E_{3}(G)$ but $P_{3} \neq F_{1}(N)=F_{2}(G) \cap N$. Lemma 2.2 applied to $G / F_{1}(G)$ yields that for some prime $p_{2} \neq p_{3}, P_{3}$ does not centralize the Sylow $p_{2}$-subgroup of $F_{2}(G) / F_{1}(G)$. Now $F_{2}(G)=F_{1}(G) C$ and $C$ is nilpotent. Thus if $S$ is the sylow $p_{2}$-subgroup of $C, S$ is invariant under $A P_{3} \ldots P_{n}$ and $\left[S, P_{3}\right] \neq F_{1}(G)$. Let $P_{2}$ be minimal 
with respect to: $P_{2} \leq S, P_{2}$ is invariant under $A P_{3} \ldots P_{n}$, and $\left[P_{2}, P_{3}\right] \notin F_{1}(G)$. Since $\left[P_{2}, P_{3}\right]$ is invariant under $A P_{3} \ldots P_{n}$ and $\left[P_{2}, P_{3}, P_{3}\right]=\left[P_{2}, P_{3}\right],[3]$, we must have $\left[P_{2}, P_{3}\right]=P_{2}$. It now only remains to choose $P_{1}$.

$P_{2} \leq F_{2}(G)$ but $P_{2} \neq F_{1}(G)$. Hence there is a prime $p_{1} \neq p_{2}$ such that $P_{2}$ does not centralize the sylow $p_{1}$-subgroup of $F_{1}(G)$. Then there is a group $p_{1}$ which is minimal with respect to: $P_{1}$ is a $P_{1}$-subgroup of $F_{1}(G), P_{1}$ is invariant under $A P_{2} \cdots P_{n}$, and $\left[P_{1}, P_{2}\right] \neq 1$. Since $\left[P_{1}, P_{2}\right]$ is invariant under $A P_{2} \ldots P_{n}$ and $\left[P_{1}, P_{2}, P_{2}\right]=\left[P_{1}, P_{2}\right] \neq 1$, we must have $\left[P_{1}, P_{2}\right]=P_{1}, P_{1}, \ldots, P_{n}$ now satisfy $(a)$ through $(g)$ and the theorem is proved.

COROLLARY 2.5. In Theorem 2.4, let $Q_{i}=P_{i} /\left(P_{i} \cap E_{i-1}(G)\right)$ for $1 \leq i \leq n$. Then $Q_{n}$ is elementary abelian and is transformed irreducibly by $A$. If $I \leq i \leq n-1$, then $Q_{i}$ is a special $p_{i}$-group and any proper oubgroups of $Q_{i}$ which are invariant under $A \prod_{i<j} P_{j}$ are contained in $Q_{i}^{\prime}$

Proof. This follows from Theorem 2.1 and from $(f)$ and $(g)$ in Theorem 2.4 .

LEMMA 2.6. Suppose $G, A, n, P_{i}$, and $p_{i}$ for $1 \leq i \leq n$ have the same meaning as in Theorem 2.4. Assume that every A-invariant proper subgroup of $G$ has nilpotent length $<n$. For $1 \leq i \leq n$, let $T_{i}=P_{i} \cap F_{i-1}(G), Q_{i}=P_{i} / T_{i}$, and $C_{i}=C_{A}\left(Q_{i}\right)$. Then $G=P_{1} P_{2} \ldots P_{n}$ and $\left[P_{j}, C_{i}\right]=1$ if $1 \leq i \leq j \leq n$.

Proof. $P_{1} P_{2} \cdots P_{n}$ is A-invariant and, from Lemma 2.3, $Z\left(P_{1} P_{2} \ldots P_{n}\right) \geq n$. Hence $P_{1} P_{2} \ldots P_{n}=G$. Let $H_{i}=C_{P_{i}}\left(C_{i}\right)$. Since $C_{i} \subseteq C, H_{i}$ is $A$-invariant. From $\left[P_{i} / T_{i}, C_{i}\right]=1$ and $\left(\left|P_{i}\right|,\left|C_{i}\right|\right)=1$ follows $H_{i} T_{i}=P_{i}$. Since $H_{n}$ is A-invariant, 
$T_{n} \leq F_{n-1}(G)$, and $P_{n} \ddagger F_{n-1}(G)$, Theorem $2.4(f)$. implies that $H_{n}=P_{n}$. Assume now $1 \leq i<n$ and $\left[P_{j}, C_{i+1}\right]=1$ if $i+1 \leq j \leq n$. $\left[P_{i}, C_{i}, P_{i+1}\right] \leq F_{i-1}(G)$ and $\left[P_{i+1}, P_{i}, C_{i}\right]=\left[P_{i}, C_{i}\right] \leq E_{i-1}(G)$. The 3 Subgroups Lemma yields $\left[P_{i+1}, C_{i}, P_{i}\right] \leq F_{i-1}(G)$. It follows from this that $\left[P_{i+1}, C_{i}\right] \leq C_{P_{i+1}}\left(Q_{i}\right)$. Let $K=C_{P_{i+1}}\left(Q_{i}\right)$. Then $K F_{i-1}(G)$ is normalized by $E_{i-1}(G) P_{i} P_{i+1} \ldots P_{n}=G$. Since $K F_{i-1}(G) / E_{i-1}(G)$ is nilpotent, we must have $K \leq F_{i}(G)$. A consequence of this is that $\left[P_{i+1}, C_{i}\right] \leq T_{i+1}$. Hence $C_{i} \leq C_{i+1}$. Then $\left[P_{j}, C_{i}\right]=1$ if $i+1 \leq j \leq n$. It follows from this that $H_{i}$ is normalized by $P_{i+1} P_{i+2} \cdots P_{n}$. Theorem $2.4(g)$ now implies that either $H_{i}=P_{i}$ or $\left[P_{i+1}, H_{i}\right] \leq E_{i-1}(G)$. Since $P_{i}=H_{i} T, T_{i} \leq E_{i-1}(G)$, and $\left[P_{i+1}, P_{i}\right] \neq F_{i-1}(G)$, we cannot have $\left[P_{i+1}, H_{i}\right] \leq E_{i-1}(G)$. Thus $H_{i}=P_{i}$ and the lemma is proved.

\section{The main results}

Throughout this section we assume $A$ is an elementary abelian group of order $p^{m}>1$ which acts as an operator group on the $p^{\prime}$-group $G$.

THEOREM 3.1. Assume $G$ is solvable and let $n=\max \left\{z\left(C_{G}(X)\right) \mid X \in A^{\#}\right\}$. If $m \geq n+2$, then $z(G)=n$.

Proof. Suppose $G$ is a counter-example of minimal order. Then if $H$ is an $A$-invariant proper subgroup of $G$, we must have $Z(H) \leq n$. Also if $H$ is an $A$-invariant non-identity normal subgroup of $G$, then $Z(G / H) \leq n$. This implies that $Z(G)=n+1$.

Let $P_{1}, \ldots, P_{n+1}$ be the A-invariant subgroups of $G$ guaranteed by Theorem 2.4. Let $T_{i}=P_{i} \cap F_{i-1}(G), Q_{i}=P_{i} / T_{i}$, and $C_{i}=C_{A}\left(Q_{i}\right)$ for $1 \leq i \leq n+1$. Now $C_{Q_{n+1}}(X)$ is $A$-invariant for $X \in A^{\#}$. Using Corollary 2.5, we see that $X \in A^{\#}$ implies $C_{Q_{n+1}}(X)=1$ or $Q_{n+1}$. 
Hence if $B_{n+1}$ is a complement to $C_{n+1}$ in $A$, we see that $\left\langle C_{Q_{n+1}}(X) \mid X \in B_{n+1}^{\#}\right\rangle=1$. Hence, by [4, Theorem 6.2.4], $B_{n+1}$ must be cyclic. This implies $\left|A: C_{n+1}\right| \leq p$.

By Lemma 2.6, $C_{i} \leq C_{i+1}$ if $I \leq i \leq n$. Let $B_{i}$ be a complement of $C_{i}$ in $C_{i+1}$. Let $X \in B_{i}^{\#}$ and $R=C_{Q_{i}}(X) . R$ is $A$-invariant and is also invariant under $C_{P_{j}}(X)=P_{j}$ for $i<j \leq n+1 . \quad\left(C_{P_{j}^{\prime}}(X)=P_{j}\right.$ from Lemma 2.6. Corollary 2.5 implies that $R$ is one of the groups $1, Q_{i}^{\prime}$, or $Q_{i} \cdot R \neq Q_{i}$ since $X \neq C_{i}$. Hence we have shown that $\left\langle C_{Q_{i}}(X) \mid X \in B_{i}^{\#}\right\rangle \leq Q_{i}^{\prime} \neq Q_{i}$. From [4, Theorem 6.2.4] it follows that $B_{i}$ is cyclic. Hence $\left|C_{i+1}: c_{i}\right| \leq p$.

From $\left|A: C_{n+1}\right| \leq p,|A| \geq p^{n+2}$, and $\left|C_{i+1}: C_{i}\right| \leq p$ for $1 \leq i \leq n$, we obtain $\left|c_{i}\right| \geq p^{i}$ for $1 \leq i \leq n$. Hence there is a non-identity element $X$ in $C_{1}$. Then Lemma 2.6 implies that $\left[P_{i}, X\right]=1$ for $I \leq i \leq n+1$. Hence $C_{G}(X)=G$. But $Z(G)=n+1$ and $l\left(C_{G}(Y)\right) \leq n$ for all $Y \in A^{\#}$. This contradiction finishes the proof.

LEMMA 3.2. Assume $m \geq 3$ and $C_{G}(X)$ is abelian for alz $X \in A^{\#}$. Then $G$ is abelian.

Proof. Let $G$ be a minimal counter-example. Then if $H$ is an $A$-invariant non-identity normal subgroup of $G, G / H$ must be abelian. It follows from this that $G^{\prime}$ is a minimal $A$-invariant normal subgroup of $G$. From [8], $G$ is nilpotent. Since $G^{\prime} \cap Z(G) \neq 1$, we must have $G^{\prime} \leq z(G)$. Then any subgroup of $G^{\prime}$ is normal in $G$. This implies that $A$ transforms $G^{\prime}$ irreducibly. Thus $C_{G^{\prime}}(X)=1$ or $=G^{\prime}$ for each $X \in A^{\#}$. Let $C=C_{A}\left(G^{\prime}\right)$ and let $B$ be a complement to $C$ in $A$. Then 
$\left\langle C_{G^{\prime}}(X) \mid X \in B^{\#}\right\rangle=1$. Hence $B$ must be cyclic and so $|C| \geq p^{2}$.

Since $C$ is not cyclic, $G=\left\langle C_{G}(X) \mid X \in C^{\#}\right\rangle$. Let $X, Y \in C^{\#}$, $H=C_{G}(X)$, and $K=C_{G}(Y) \cdot H$ and $K$ are both abelian and

$[H, K,\langle X\rangle] \leq\left[G^{\prime},\langle X\rangle\right]=1$ and $[\langle X\rangle, H, K]=1$. The 3 Subgroups Lemma implies $[K,\langle X\rangle, H]=1$. Now $K$ is $A$-invariant and so $K=[K,(X)] C_{K}(X)$. But $C_{K}(X) \leq H$ and $H$ is abelian. Thus $[K, H]=[K,\langle X\rangle, H]=1$. It follows that $G$ is abelian.

THEOREM 3.3. Assume $m \geq 4, G$ is solvable, and $C_{G}(X)$ is supersolvable for all $X \in A^{\#}$. Then $G$ is supersolvable.

Proof. Suppose $G$ is a counter-example of minimal order. If $B$ is an $A$-invariant non-identity normal subgroup of $G$, then $G / H$ is supersolvable. It follows from this that $D(G)=1$ and there is only one minimal $A$-invariant normal subgroup of $G$. Therefore $F(G)$ is an elementary abelian $q$-group for some prime $q$. From Theorem 2.1, $Z(G)=2$. Hence $G / F(G)$ is a nilpotent $q^{\prime}$-group. Now if $G / F(G)$ were abelian of exponent dividing $q-1$, then from [1, Theorem 6.1], $G$ would be supersolvable. Thus for some prime $r \neq q$, there is an $A$-invariant r-subgroup $R$ in $G$ such that either $R$ is non-abelian or the exponent of $R$ does not divide $q-1$. Then $R F(G)$ is an $A$-invariant subgroup of $G$ and $R F(G)$ is not supersolvable. Thus $R F(G)=G$.

Let $C=C_{A}(R)$ and let $B$ be a complement to $C$ in $A \cdot I$ assert that $|B| \geq p^{3}$. Suppose to the contrary that $|B|<p^{3}$. Then $|C| \geq p^{2}$ and so $F(G)=\left\langle C_{F(G)}(X) \mid X \in C^{\#}\right\rangle$. Thus there would be an $X \in C^{\#}$ such that $C_{F(G)}(X) \neq 1$. Now $C_{R}(X)=R$ and $A$ is abelian. Thus $C_{F(G)}(X)$ is invariant under $A R$. By Maschke's Theorem, there is an $A R$-invariant complement $K$ to $C_{F(G)}(X)$ in $F(G)$. Since $F(G)$ is abelian, $K$ and $C_{F(G)}(X)$ are normal in $R F(G)=G$. Since there is only one minimal $A$-invariant normal subgroup in $G$, we must have $K=1$. Then $C_{F(G)}(X)=F(G)$ which implies $G=F(G) R=C_{G}(X)$ is supersolvable. 
Thus $|B| \geq p^{3}$. Now let $X \in B^{\#}, C_{R}(X) \neq R$ and so $C_{R}(X) F(G)$ is a proper A-invariant subgroup of $G$. Thus $C_{R}(X) F(G)$ must be supersolvable. It follows from this that $C_{R}(X)$ is abelian of exponent dividing $(q-1)$. Lemma 3.2 now implies that $R$ is abelian. Since $R=\left\langle C_{R}(X) \mid X \in B^{\not}\right\rangle$, the exponent of $R$ must divide $(q-1)$ and the theorem is proved.

\section{Examples}

1. Let $A$ be an elementary abelian $p$-group of order $p^{n+1}$ where $n \geq 1$. Then by [5], there is an odd order $p^{\prime}$-group $G$ on which $A$ operates in a fixed-point-free manner and such that $Z(G)=n+1$. If $X \in A^{\#}$, then $C_{G}(X)^{\prime}$ admits a fixed-point-free abelian operator group of order $p^{n}$. By [7], this implies that $l\left(C_{G}(X)\right) \leq n$. Hence the requirement $m \geq n+2$ is necessary in Theorem 2.1.

2. Let $G$ be a non-abelian group of order 27 and exponent 3 . Let $a$ and $b$ be any elements generating $G$. Then there are automorphisms $x$ and $y$ of $G$ such that $a^{x}=a, b^{x}=b^{-1}$, $a^{y}=a^{-1}$, and $b^{y}=b . x$ and $y$ generate an elementary abelian group $A$ of order $4 \cdot C_{G}(z)$ has order 3 for all $z \in A^{\#}$ but $G$ is not abelian. Thus the requirement $m \geq 3$ is necessary in Lemma 3.2.

3. Let $p, q, r$, and $s$ be four distinct odd primes such that $q \equiv 1(\bmod r s)$ and $r \equiv I(\bmod s)$. (For example, $p=5, q=43$, $r=7$, and $s=3$ would be satisfactory.) Let $A$ be elementary abelian of order $p^{3}$. Using the methods of [5], it is possible to construct a solvable group $G$ such that:

(a) $A$ acts in a fixed-point-free manner on $G$;

(b) $Z(G)=3$;

(c) $F_{1}(G)$ is an elementary abelian q-group;

(d) $F_{2}(G) / F_{1}(G)$ is an elementary abelian r-group; 
(e) $G / F_{2}(G)$ is an elementary abelian s-group.

Now if $X \in A^{\#}$, then $C_{G}(X)$ admits a fixed-point-free operator group of order $p^{2}$. Thus, by [6], $l\left(C_{G}(X)\right) \leq 2$. From $(c),(d)$, and (e), it follows that $C_{G}(X)$ is supersolvable. However, $Z(G)=3$; and so $G$ is not supersolvable. Thus $m \geq 4$ is necessary in Theorem 3.3 .

\section{References}

[1] Roger Carter and Trevor Hawkes, "The F-normalizers of a finite soluble group", J. AZgebra 5 (1967), 175-202.

[2] Walter Feit and John G. Thompson, "Solvability of groups of odd order", Pacific J. Math. 13 (1963), 775-1029.

[3] George Glauberman, "Fixed points in groups with operator groups", Math. Z. 84 (1964), 120-125.

[4] Daniel Gorenstein, Finite groups (Harper and Row, New York, Evanston, London, 1968).

[5] Fletcher Gross, "A note on fixed-point-free solvable operator groups", Proc. Amer. Math. Soc. 19 (1968), 1363-1365.

[6] P. Hall and Graham Higman, "On the p-length of p-soluble groups and reduction theorems for Burnside's problem", Proc. London Math. Soc. (3) $6(1956), 1-42$.

[7] Ernest E. Shult, "On groups admitting fixed point free abelian operator groups", IZlinois J. Math. 9 (1965), 701-720.

[8] J.N. Ward, "On finite groups admitting automorphisms with nilpotent fixed-point group", Bull. Austral. Math. Soc. 5 (1971), 281-282.

[9] J.N. Ward, "On finite soluble groups and the fixed-point groups of automorphisms", BulZ. Austral. Math. Soc. 5 (1971), 375-378.

University of Utah,

Salt Lake City,

Utah, USA. 